Schutte, R., Whincup, P. H., Papacosta, O., Lennon, L. T., Macfarlane, P. W. and Wannamethee, G. (2017) Liver enzymes are not directly involved in atrial fibrillation: a prospective cohort study. European Journal of Clinical Investigation, 47(8), pp. 583-590.

(doi:10.1111/eci.12779)

There may be differences between this version and the published version. You are advised to consult the publisher's version if you wish to cite from it.

This is the peer-reviewed version of the following article: Schutte, R., Whincup, P. H., Papacosta, O., Lennon, L. T., Macfarlane, P. W. and Wannamethee, G. (2017) Liver enzymes are not directly involved in atrial fibrillation: a prospective cohort study. European Journal of Clinical Investigation, 47(8), pp. 583-590, which has been published in final form at 10.1111/eci.12779. This article may be used for noncommercial purposes in accordance with Wiley Terms and Conditions for Self-Archiving.

http://eprints.gla.ac.uk/143723/

Deposited on: 06 July 2017

Enlighten - Research publications by members of the University of Glasgow http://eprints.gla.ac.uk 


\section{Liver enzymes are not directly involved in atrial fibrillation: a prospective cohort study}

Running head: Atrial fibrillation and liver enzymes

Rudolph Schutte, PhD ${ }^{\text {b,c,d; }}$ Peter H Whincup, MD, Ph D ${ }^{\text {e }}$; Olia Papacosta, MSc ${ }^{\text {a }}$ Lucy T Lennon, MSc ${ }^{\mathrm{a}}$; Peter W Macfarlane, DSc ${ }^{\mathrm{f}}$; Goya Wannamethee, $\mathrm{PhD}^{\mathrm{a}}$

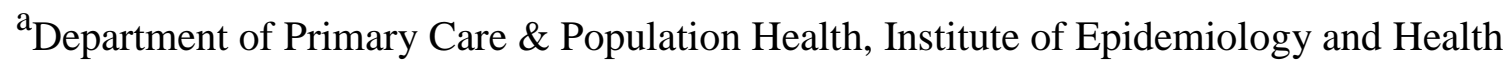
Care, University College London Medical School, London, UK; ${ }^{b}$ Hypertension in Africa Research Team (HART), North-West University, Potchefstroom, South Africa; ${ }^{\mathrm{c}}$ South African Medical Research Council: Unit for Hypertension and Cardiovascular Disease, North-West University, Potchefstroom, South Africa; ${ }^{\mathrm{d}}$ Department of Medicine and Healthcare Science, Faculty of Medical Science, Anglia Ruskin University, Chelmsford, UK;

'Population Health Research Institute, St George's, University of London, London, UK; ${ }^{\mathrm{f}}$ Institute of Cardiovascular and Medical Sciences, University of Glasgow, Glasgow, UK

Manuscript 2454; references 34; tables 3; figures 1;

\section{Correspondence:}

Dr. Rudolph Schutte, $\mathrm{PhD}$

Department of Medicine and Healthcare Science, Faculty of Medical Science

Anglia Ruskin University

Chelmsford CM1 1SQ

Telephone: +44 (0) 1245493035

e-mail: $\quad$ rudolph.schutte@anglia.ac.uk 


\begin{abstract}
Background Epidemiological evidence proposes the direct involvement of the liver enzymes in atrial fibrillation. These relationships are controversial and mechanistically unclear. As part of the British Regional Heart Study, we investigated whether change in liver enzymes over time associates with atrial fibrillation in men initially free of this heart condition.
\end{abstract}

Materials and Methods We prospectively investigated change (delta) in liver enzymes and new-onset atrial fibrillation in a representative sample of 1428 men aged 60-79 years.

Results Mean follow-up was 12.3 years, after which 108 new atrial fibrillation cases were identified. The liver enzymes did not differ at baseline or follow-up, except for gammaglutamyl transferase which was higher at follow-up in men who developed atrial fibrillation compared to those who did not $(P<0.0001)$. Change in GGT was greater in men who developed AF than those who did not (+6.12 vs. $-2.60 \mathrm{U} / 1, P=0.036)$. N-terminal pro-brain natriuretic peptide (baseline and follow-up, $P<0.0001$ ) and total bilirubin (follow-up only, $P<0.0001)$ were also higher in men who developed atrial fibrillation while serum haemoglobin was similar at baseline and follow-up ( $\mathrm{P} \geq 0.74)$. Atrial fibrillation was associated with change in gamma-glutamyl transferase (OR, 1.18; 95\%CI, 1.01-1.37) after multiple adjustments and exclusions. However, after adjusting for baseline $(P=0.088)$ or change $(P=0.40)$ in $\mathrm{N}$-terminal pro-brain natriuretic peptide, the association between atrial fibrillation and change in gamma-glutamyl transferase was lost.

Conclusion The direct relationship between atrial fibrillation and liver enzymes is absent and depends, at least in part, on the progression of heart failure as captured by N-terminal pro-brain natriuretic peptide.

Keywords: Liver enzymes, atrial fibrillation, heart failure 


\section{Introduction}

The direct involvement of liver enzymes in cardiovascular disease, especially gammaglutamyl transferase (GGT) [1], remains controversial [2,3]. Recent population-based evidence suggests that circulating liver enzymes also associates independently with atrial fibrillation (AF) [4-7]. The evidence on this finding is limited, inconsistent and mechanistically unclear. In the Framingham cohort, associations existed between AF risk and alanine transaminase (ALT) and aspartate transaminase (AST) [6], while in the Atherosclerosis Risk in Communities study, GGT but not ALT or AST predicted new-onset AF [7]. These studies [6,7] mostly accounted for baseline characteristics of participants initially free of AF, rendering it difficult to capture and account for the evolving pathophysiology. Heart failure contributes to atrial dysfunction and vice versa [8-11], but also increases central- and hepatic venous pressure leading to congestive hepatopathy, augmenting circulating GGT, bilirubin and alkaline phosphatase (ALP) levels [12-14]. However, the relationship between AF and liver enzymes could be more complex as the association also exists in the absence of clinically diagnosed heart failure [6]. Currently, no evidence is available on the change in liver enzymes over time and new-onset AF. With the incidence of AF projected to more than double by 2030 with significant economic implications [15], an understanding of the mechanisms at work has clinical importance. To investigate the relationship between new-onset $\mathrm{AF}$ and change in liver enzymes over time, we used prospective data from the British Regional Heart Study.

\section{Materials and Methods}

Reporting of this study conforms to STROBE statement [16].

\section{Study population}

The British Regional Heart Study is a prospective cohort study involving 7735 randomly selected men from 24 towns representing all major British regions (response rate, 78\%) [17]. 
We followed the men since 1978-1980 when they were aged 40-59 years and re-examined them at ages 60-79 years (1998-2000) and 71-92 years (2010-2012). The first reexamination after 20 years included 4252 men ( $77 \%$ response rate) and the second after 32 years 1722 men (55\% response rate). For the present investigation, we used the first reexamination as baseline (1998-2000) and the second (2010-2012) as the follow-up for outcome assessment. All participants gave informed written consent and could withdraw at any stage. The protocol complied with the Declaration of Helsinki of 1975 (as revised in 1983) and all relevant local research ethics committees provided ethical approval.

\section{Participant examinations and exclusions}

The re-examinations included completion of a questionnaire on lifestyle and medical history, a physical examination, a 12-lead electrocardiogram (ECG), and collection of fasting blood samples using the Sarstedt Monovette System for biochemical analysis.

Electrocardiograms were recorded using a Siemens Sicard 460 and transmitted to the University of Glasgow ECG core lab for analysis using the University of Glasgow ECG interpretation program [18] with an additional model for automated Minnesota Coding [19]. All reports were carefully reviewed and edited wherever necessary. Prevalent heart failure at baseline and follow-up was based on a doctor-confirmed diagnosis of heart failure from medical records and verified by review of available clinical information from primary and secondary care records.

Blood samples were frozen and stored at $-20^{\circ} \mathrm{C}$ on the day of collection and transferred in batches for storage at $-70^{\circ} \mathrm{C}$ until analysis, which was carried out after no more than one freeze-thaw cycle. Details of measurement and classification methods for smoking status, alcohol intake, blood pressure, and blood lipids in this cohort have previously been described [20-22]. C-reactive protein (CRP) was assayed by ultrasensitive nephelometry (Dade 
Behring, Milton Keynes, United Kingdom) and plasma glucose by a glucose oxidase method using a Falcor-600 automated analyzer. Hepatic enzymes including GGT, AST, ALT and ALP, as well as serum albumin, total protein and total bilirubin, were measured using a Hitachi 747 automated analyzer. The normal laboratory range was 8 to 61 U/l for GGT, <37 U/l for AST, <41 U/1 for ALT and 35-129 U/1 for ALP. The measurement of baseline and follow-up liver enzymes followed the same enzymatic calorimetric assay principle [23-25]. $\mathrm{N}$-terminal prohormone of brain natriuretic peptide (NT-proBNP) was measured using the Elecsys 2010 electrochemiluminescence method (Roche Diagnostics, Burgess Hill, UK).

\section{Statistical analysis}

For database management and statistical analysis, we used SAS software, version 9.4 (SAS Institute Inc., Cary, NC). The distributions of GGT, ALT, AST, ALP, blood glucose, NTproBNP, and C-reactive protein were normalized by logarithmic transformation. We compared means and proportions by the standard normal z-test and the $\chi^{2}$ statistic, while longitudinal changes in means and proportions were assessed by the PROC MIXED procedure and McNemar's test, respectively. Statistical significance was set at a level of 0.05 on 2-sided tests. We analyzed associations with AF using logistic regression analysis to calculate odds ratios (OR) standardized to 1 standard deviation (SD) and 95\% confidence intervals (CI), while allowing for covariates and potential confounders. We applied the forward stepwise procedure with $P$ values for explanatory variables to enter and stay in the model set at 0.05 . Covariates included in the model were respective baseline liver enzymes, baseline age, body mass index, systolic blood pressure, total cholesterol, serum glucose, physical activity, smoking, drinking, use of anti-hypertensive medication, and follow-up time. Physical activity used in the regression models was classified into inactive, occasional, light, moderate and vigorous; smoking into never, former and current smokers; and drinking into none, occasional, light, moderate, and heavy drinkers. We performed sensitivity analysis 
by additionally, but separately, adjusting for baseline C-reactive protein, ALT, AST, ALT/AST ratio, bilirubin, NT-proBNP and their respective change $(\Delta)$. We also repeated our analysis after excluding participants with GGT outside its reference range (>61 U/l), and those who had a previous myocardial infarction or heart failure at baseline or follow-up. Lastly, since NT-proBNP levels above $900 \mathrm{pg} / \mathrm{ml}$ in people aged 50-75 years is indicative of heart failure [26,27], we repeated our analysis in participants who had NT-proBNP levels below $900 \mathrm{pg} / \mathrm{ml}$ at both baseline and follow-up.

\section{Results}

\section{Participant characteristics}

We based our study on 1722 men who took part in both the first and second follow-up examinations. We excluded 256 participants without blood measurements at baseline or follow-up, 20 with no electrocardiogram measurements at follow-up, and 18 with AF at baseline, leaving 1428 participants for inclusion in the analysis. After a mean follow-up of 12.3 years (5th to 95th percentile interval, 12.1-12.9 years), 108 new cases of AF were identified. The baseline characteristics of participants who developed or did not develop AF are presented in Table 1 and follow-up characteristics in Supplementary Table 1. Circulating levels of AST, ALT and ALP at baseline ( $P \geq 0.083)$ and follow-up $(P \geq 0.054)$ did not differ between men who did or did not develop AF (Supplementary Figure 1). GGT was also similar in both groups at baseline $(P=0.93)$, however GGT at follow-up $(P<0.0001$, Figure 1A) and change $(\Delta)$ in GGT from baseline to follow-up (+6.12 vs. $-2.60 \mathrm{U} / 1, P=0.036)$ were higher in the men with AF at follow-up. In addition, both NT-proBNP $(P<0.0001$, Figure 1B) and total bilirubin $(P<0.0001$, Figure 1C) at follow-up were higher in the AF group and increased more markedly from baseline to follow-up (both $\mathrm{P}<0.0001$ ), while mean haemoglobin did not differ between the groups at both baseline $(P=0.82)$ and follow-up $(P=0.74$, Figure 1D). 
Self-reported alcohol intake did not differ between the groups at baseline $(P=0.55)$ or follow-up $(P=0.62)$ and intake declined from baseline to follow-up in both the $\mathrm{AF}(P=0.028)$ and non-AF groups $(P<0.0001$; data not shown). Similarly, the AST/ALT ratio as a measure of alcoholic liver damage did not differ between the groups at baseline $(P=0.18)$ or follow-up $(P=0.21)$ and decreased in both groups from baseline to follow-up (with AF, $P<0.0001$; without AF, $P<0.0001$; data not shown).

\section{Univariate and multivariate analyses}

In univariate analysis in the total group (Supplementary Table 2), $\Delta$ NT-proBNP correlated with $\Delta$ GGT $(\mathrm{r}=0.14, P<0.0001), \Delta$ total bilirubin $(\mathrm{r}=0.13, P<0.0001)$ and $\Delta$ ALP $(\mathrm{r}=0.10$, $P=0.0001)$, but not with $\triangle \mathrm{ALT}(\mathrm{r}=0.01, \mathrm{P}=0.61)$ or $\triangle \mathrm{AST}(\mathrm{r}=0.04, P=0.17)$.

In univariate logistic regression analysis (Table 2), none of the liver enzymes at baseline, or the change in AST, ALT or ALP was associated with AF. However a 1-SD increase in $\Delta$ GGT was associated with a $21.7 \%(95 \% \mathrm{CI}, 5.2-40.6 \% ; P=0.008)$ increased odds of developing AF. After adjusting for baseline GGT and other covariates and potential confounders (Table 2), the above association between AF and $\Delta$ GGT remained significant (OR, 1.18; 95\% CI, 1.01-1.37; $P=0.032)$. Associations with AST, ALT and ALP $(P \geq 0.14)$ or their change $(P \geq 0.73)$ remained non-significant.

\section{Sensitivity analysis}

Additional adjustments (Table 3) for baseline C-reactive protein as a marker of inflammation $(P=0.018), \operatorname{ALT}(P=0.032), \operatorname{AST}(P=0.031)$, the AST/ALT ratio to account for alcoholic liver disease $(P=0.032)$, ALP $(P=0.032)$ as well as their respective change from baseline to followup $(P \leq 0.032)$, did not appreciably affect our findings. Our results also remained significant after excluding those with GGT levels outside the reference range ( $>61 \mathrm{U} / 1, P=0.0004)$, those with a previous myocardial infarction $(P=0.032)$, and even after excluding 74 participants 
with prevalent and incident heart failure $(P=0.015)$. However, when accounting for baseline $(P=0.088)$ or change in NT-proBNP $(P=0.40)$ as a clinical measure of heart failure and left ventricular systolic- and diastolic dysfunction, the relationship between AF risk and $\Delta \mathrm{GGT}$ was no longer present. When adjusting for $\Delta$ total bilirubin, statistical significance was also lost $(P=0.13)$. Entering both $\triangle \mathrm{NT}$-proBNP and $\Delta$ total bilirubin in the model confirmed the absence of an independent relationship between AF and $\Delta$ GGT $(P=0.62)$. Lastly, by repeating our analysis in participants with NT-proBNP levels below $900 \mathrm{pg} / \mathrm{ml}$ at both baseline and follow-up, the relationship between AF and $\Delta \mathrm{GGT}$ was absent (OR, 0.86; 95\% CI, $0.55-1.33 ; P=0.50)$.

\section{Discussion}

We investigated in a representative sample of older British men initially free of AF, whether change in liver enzymes over 12.3 years were associated with new-onset AF. While no evidence existed for baseline liver enzymes, initial findings indicated a seemingly robust association between AF and change in GGT after multiple adjustments and exclusions including participants with prevalent and incident heart failure. However, this relationship was dependent on the progression of heart failure as represented by change in NT-proBNP.

The concept of cardiovascular events, and more recently AF relating to liver enzymes has become a matter of debate and remains mechanistically unclear [1-7]: Sinner et al. [6] followed 3,744 participants (mean age, 65 years) free of AF and heart failure at baseline and found a respective $19 \%$ and $12 \%$ increased risk of developing new-onset AF per one standard deviation increase in baseline ALT and AST. The authors confirmed these results after multiple adjustments including interim heart failure as a dichotomous variable, but not for AST after adjusting for C-reactive protein as a marker of systemic inflammation [6]. They provided no descriptive data on interim heart failure and incidence was likely higher in the AF group that was older by a mean of 6.8 years [6]. In our study population of similar age, 
no such evidence existed for the liver transaminases, even in univariate analysis and including participants with incident heart failure. Alonso et al. provided the first prospective evidence for linking new-onset AF with GGT, but were unable to confirm a relationship with ALT or AST in 9,333 men and women aged 53-75 years [7]. This study involved extremecase statistical analysis by quintiles of baseline GGT. The authors did include baseline NTproBNP and C-reactive protein in the quintile analysis, and confirmed the results after excluding participants with prevalent heart failure at baseline. However, there were no indication whether NT-proBNP was included in the continuous analysis when mentioning a $20 \%$ increased risk of AF per doubling of GGT [7].

The above studies were limited in their ability to capture the pathophysiological progression of disease by accounting only for baseline covariates and potential confounders. We were able to show in those who developed AF, that a greater increase in GGT was paralleled by NT-proBNP and bilirubin as respective sensitive markers of progressive heart failure [28] and congestive hepatopathy [29]. In heart failure patients, elevated liver function tests are a consequence of either backward failure, increasing central venous pressure; or forward failure with attenuated cardiac output and consequent liver perfusion [12-14,30]. Decreased liver perfusion elevates the transaminases due to hepatocellular damage $[14,29,31]$, while increased central venous pressure transmits to the hepatic veins and results in elevations in GGT, bilirubin and ALP [32,33]. In our study, GGT and bilirubin, which did not differ at baseline between the groups, were higher in those with AF at follow-up, while ALP was borderline higher in the AF group at baseline and follow-up. ALP is normally elevated in more extreme cholestatic conditions [34] and it is reasonable to assume that the effects of hepatic congestion would firstly be seen for GGT and bilirubin before the impediment to bile flow. Van Deursen et al. [14] reported that abnormalities in all liver function tests were associated with invasively-measured central venous pressure. 
Importantly, higher central venous pressure was predominantly related to GGT and bilirubin [14]. From our results, it seems more likely that the backward failure-central venous pressure mechanism could explain our findings, especially since the liver transaminases did not differ between those with and without AF. In addition, the positive univariate associations in the total group of NT-proBNP with $\Delta \mathrm{GGT}, \Delta \mathrm{ALP}$ and $\Delta$ total bilirubin, but not with $\triangle \mathrm{ALT}$ or $\triangle \mathrm{AST}$, at least in part, supports this pathway.

The link between AF and $\Delta \mathrm{GGT}$ was dependent on $\Delta \mathrm{NT}$-proBNP, even in the absence of diagnosed heart failure, therefore capturing the confounding effect of heart failure at subclinical level. Supporting this was the absence of the AF- $\Delta$ GGT link when repeating our analysis in participants who consistently had NT-proBNP levels below $900 \mathrm{ng} / \mathrm{ml}$ (above which is indicative of heart failure for people aged 50-75 years) [26,27]. Heart failure and AF often coincide with causal pathways in both directions [8-11]. This is at least partly supported by our results confirming the known $[35,36]$ relationship between NT-proBNP and AF (OR, 1.53; 95\% CI, 1.24-1.88; $P<0.0001)$ in fully adjusted models which also included $\Delta \mathrm{GGT}$ and $\Delta$ total bilirubin. On balance, the link between the failing heart and the liver described above also seems evident with the disappearance of the association between AF and GGT after adjusting for $\Delta$ total bilirubin as both GGT and bilirubin are elevated in congestive hepatopathy [12-14]. Taken together, no direct relationship seems apparent between AF and the liver enzymes, which, even in the absence of prevalent and incident heart failure, seems due to progression of heart failure leading to both AF and hepatic congestion.

The current study must be interpreted within the context of its potential limitations. Although our cohort is socially representative of older white British men, our results may not be generalizable to younger populations, women and other ethnicities. We investigated overall AF only and therefore cannot comment on the associations of the liver enzymes and AF type (i.e., valvular, paroxysmal, persistent, or permanent). The increase in total bilirubin 
from baseline to follow-up could also be due to augmented bilirubin production (increased haemolysis) [37], however this seems unlikely since haemoglobin levels did not differ between those with and without AF at both baseline and follow-up. Although the same enzymatic calorimetric assay principle was used to determine the liver enzyme levels at baseline and follow-up, the storage time difference of samples could have affected the results. However, a similar trend in GGT levels could be seen in participants who passed away between after 1998-2000 (not included in the present study) with GGT being higher in those with AF compared to those without (Supplementary Figure 2).

In conclusion, the direct relationship between atrial fibrillation and liver enzymes is absent and depends, at least in part, on the progression of heart failure as captured by NT-proBNP. 


\section{Acknowledgements}

The British Regional Heart Study is a British Heart Foundation (BHF) research group and receives support from BHF Programme Grant (RG/08/013/25942). The examinations of study men were supported by British Heart Foundation Project Grants PG97012 and PG/09/024.

\section{Conflict of Interest}

None declared 


\section{References}

1. Kunutsor SK, Apekey TA, Khan H. Liver enzymes and risk of cardiovascular disease in the general population: a meta-analysis of prospective cohort studies. Atherosclerosis 2014;236:7-17.

2. Kunutsor SK, Bakker SJ, Kootstra-Ros JE, Gansevoort RT, Dullaart RP. Circulating gamma glutamyltransferase and prediction of cardiovascular disease. Atherosclerosis 2015;238:356-64.

3. Ndrepepa G, Braun S, Cassese S, Fusaro M, Laugwitz KL, Schunkert H, et al. Relation of Gamma-Glutamyl Transferase to Cardiovascular Events in Patients With Acute Coronary Syndromes. Am J Cardiol 2016.

4. Markus MR, Meffert PJ, Baumeister SE, Lieb W, Siewert U, Schipf S, et al. Association between hepatic steatosis and serum liver enzyme levels with atrial fibrillation in the general population: The Study of Health in Pomerania (SHIP). Atherosclerosis 2016;245:123-31.

5. Targher G, Mantovani A, Pichiri I, Rigolon R, Dauriz M, Zoppini G, et al. Nonalcoholic fatty liver disease is associated with an increased prevalence of atrial fibrillation in hospitalized patients with type 2 diabetes. Clin Sci (Lond) 2013;125:301-9.

6. Sinner MF, Wang N, Fox CS, Fontes JD, Rienstra M, Magnani JW, et al. Relation of circulating liver transaminase concentrations to risk of new-onset atrial fibrillation. Am $\mathbf{J}$ Cardiol 2013;111:219-24.

7. Alonso A, Misialek JR, Amiin MA, Hoogeveen RC, Chen LY, Agarwal SK, et al. Circulating levels of liver enzymes and incidence of atrial fibrillation: the Atherosclerosis Risk in Communities cohort. Heart 2014;100:1511-6.

8. Kurt M, Wang J, Torre-Amione G, Nagueh SF. Left atrial function in diastolic heart failure. Circ Cardiovasc Imaging 2009;2:10-5. 
9. Patel DA, Lavie CJ, Milani RV, Gilliland Y, Shah S, Ventura HO. Association of Left Ventricular Geometry With Left Atrial Enlargement in Patients With Preserved Ejection Fraction. Congest Heart Fail 2012;18:4-8.

10. Larned JM, Raja Laskar S. Atrial fibrillation and heart failure. Congest Heart Fail 2009; 15:24-30.

11. Lubitz SA, Benjamin EJ, Ellinor PT. Atrial fibrillation in congestive heart failure. Heart Fail Clin 2010;6:187-200.

12. Auer J. What does the liver tell us about the failing heart? Eur Heart J 2013;34:711-4.

13. Samsky MD, Patel CB, DeWald TA, Smith AD, Felker GM, Rogers JG, et al. Cardiohepatic interactions in heart failure: an overview and clinical implications. J Am Coll Cardiol 2013;61:2397-405.

14. van Deursen VM, Damman K, Hillege HL, van Beek AP, van Veldhuisen DJ, Voors AA. Abnormal liver function in relation to hemodynamic profile in heart failure patients. $\mathrm{J}$ Card Fail 2010;16:84-90.

15. Sheikh A, Patel NJ, Nalluri N, Agnihotri K, Spagnola J, Patel A, et al. Trends in hospitalization for atrial fibrillation: epidemiology, cost, and implications for the future. Prog Cardiovasc Dis 2015;58:105-16.

16. Simera I, Moher D, Hoey J, Schulz KF, Altman DG. A catalogue of reporting guidelines for health research. Eur J Clin Invest 2010;40:35-53.

17. Lennon LT, Ramsay SE, Papacosta O, Shaper AG, Wannamethee SG, Whincup PH. Cohort Profile Update: The British Regional Heart Study 1978-2014: 35 years follow-up of cardiovascular disease and ageing. Int J Epidemiol 2015;44:826-.

18. Macfarlane PW, Devine B, Clark E. The University of Glasgow (Uni-G) ECG Analysis Program. Computers in Cardiology 2005;32:451-4. 
19. Macfarlane PW, Latif S. Automated serial ECG comparison based on the Minnesota code. J Electrocardiol 1996;29 Suppl:29-34.

20. Emberson JR, Whincup PH, Walker M, Thomas M, Alberti KG. Biochemical measures in a population-based study: effect of fasting duration and time of day. Ann Clin Biochem 2002;39:493-501.

21. Wannamethee SG, Lowe GD, Whincup PH, Rumley A, Walker M, Lennon L. Physical activity and hemostatic and inflammatory variables in elderly men. Circulation 2002;105:1785-90.

22. Wannamethee SG, Whincup PH, Shaper AG, Rumley A, Lennon L, Lowe GD. Circulating inflammatory and hemostatic biomarkers are associated with risk of myocardial infarction and coronary death, but not angina pectoris, in older men. $\mathrm{J}$ Thromb Haemost 2009;7:1605-11.

23. Szasz G. New substrates for measuring gamma-glutamyl transpeptidase activity. Z Klin Chem Klin Biochem 1974;12:228.

24. Persijn JP, van der Slik W. A new method for the determination of gammaglutamyltransferase in serum. J Clin Chem Clin Biochem 1976;14:421-7.

25. Tietz NW. Fundamentals of clinical chemistry. 3rd ed: WB Saunders; 1986.

26. Daniels LB, Maisel AS. Natriuretic peptides. J Am Coll Cardiol 2007;50:2357-68.

27. Maisel A, Mueller C, Adams K, Jr., Anker SD, Aspromonte N, Cleland JG, et al. State of the art: using natriuretic peptide levels in clinical practice. Eur J Heart Fail 2008;10:824-39.

28. Roberts E, Ludman AJ, Dworzynski K, Al-Mohammad A, Cowie MR, McMurray JJ, et al. The diagnostic accuracy of the natriuretic peptides in heart failure: systematic review and diagnostic meta-analysis in the acute care setting. BMJ 2015;350:h910. 
29. Giannini EG, Testa R, Savarino V. Liver enzyme alteration: a guide for clinicians. CMAJ 2005;172:367-79.

30. Makarewicz-Wujec M, Kozlowska-Wojciechowska M. Nutrient intake and serum level of gamma-glutamyltransferase, MCP-1 and homocysteine in early stages of heart failure. Clin Nutr 2011;30:73-8.

31. Seeto RK, Fenn B, Rockey DC. Ischemic hepatitis: clinical presentation and pathogenesis. Am J Med 2000;109:109-13.

32. Poelzl G, Ess M, Mussner-Seeber C, Pachinger O, Frick M, Ulmer H. Liver dysfunction in chronic heart failure: prevalence, characteristics and prognostic significance. Eur J Clin Invest 2012;42:153-63.

33. Lau GT, Tan HC, Kritharides L. Type of liver dysfunction in heart failure and its relation to the severity of tricuspid regurgitation. Am J Cardiol 2002;90:1405-9.

34. Moss DW. Physicochemical and pathophysiological factors in the release of membrane-bound alkaline phosphatase from cells. Clin Chim Acta 1997;257:133-40.

35. Pilatis ND, Anyfantakis ZA, Spiliopoulos K, Degiannis D, Chaidaroglou A, Vergou G, et al. The Role of BNP and CRP in Predicting the Development of Atrial Fibrillation in Patients Undergoing Isolated Coronary Artery Bypass Surgery. ISRN Cardiol 2013;2013:235018.

36. Jug B, Sebestjen M, Sabovic M, Pohar M, Keber I. Atrial fibrillation is an independent determinant of increased NT-proBNP levels in outpatients with signs and symptoms of heart failure. Wien Klin Wochenschr 2009;121:700-6.

37. Fevery J, Blanckaert N. What can we learn from analysis of serum bilirubin? J Hepatol 1986;2:113-21. 


\section{Legend}

Figure 1. Comparison of gamma-glutamyl transferase, N-terminal prohormone of brain natriuretic peptide (NT-proBNP), total bilirubin and haemoglobin at baseline and follow-up between those with and without atrial fibrillation at follow-up. AF, atrial fibrillation. 
Table 1 Baseline characteristics of men with and without atrial fibrillation (AF) at followup

\begin{tabular}{|c|c|c|c|}
\hline & $\begin{array}{l}\text { Without AF } \\
(n=1320)\end{array}$ & $\begin{array}{l}\text { With AF } \\
(n=108)\end{array}$ & $\boldsymbol{P}$ \\
\hline Age (years) & $66.1 \pm 4.6$ & $67.9 \pm 5.2$ & 0.001 \\
\hline Body mass index $\left(\mathrm{kg} / \mathrm{m}^{2}\right)$ & $26.6 \pm 3.3$ & $27.2 \pm 3.3$ & 0.10 \\
\hline Systolic blood pressure $(\mathrm{mmHg})$ & $145.5 \pm 21.8$ & $149.8 \pm 24.7$ & 0.12 \\
\hline Diastolic blood pressure $(\mathrm{mmHg})$ & $85.0 \pm 10.2$ & $85.3 \pm 10.1$ & 0.21 \\
\hline Gamma-glutamyl transferase (U/l) & $27.8(13.0-80.0)$ & $27.9(13.0-94.0)$ & 0.93 \\
\hline Aspartate transaminase (U/l) & $23.5(16.0-35.0)$ & $22.9(16.0-38.0)$ & 0.36 \\
\hline Alanine transaminase (U/l) & $16.7(9.0-33.5)$ & $15.6(8.0-34.0)$ & 0.12 \\
\hline Alkaline phosphatase (U/l) & $78.3(51.0-119.5)$ & $81.4(56.0-117.0)$ & 0.083 \\
\hline ALT:AST & $1.48 \pm 0.50$ & $1.54 \pm 0.54$ & 0.18 \\
\hline Total bilirubin $(\mu \mathrm{mol} / \mathrm{l})$ & $9.11(4.00-17.00)$ & $9.70(5.00-18.00)$ & 0.22 \\
\hline Total protein $(\mathrm{g} / \mathrm{l})$ & $76.6 \pm 4.3$ & $76.4 \pm 4.5$ & 0.53 \\
\hline Serum albumin $(\mathrm{g} / \mathrm{l})$ & $44.5 \pm 2.6$ & $43.7 \pm 2.7$ & 0.004 \\
\hline Blood glucose $(\mathrm{mmol} / \mathrm{l})$ & $5.74(4.70-7.40)$ & $5.73(4.80-7.10)$ & 0.60 \\
\hline Total cholesterol (mmol/l) & $6.09 \pm 1.06$ & $6.17 \pm 1.09$ & 0.46 \\
\hline C-reactive protein (mg/l) & $1.31(0.23-8.10)$ & $1.73(0.33-12.2)$ & 0.013 \\
\hline NT-proBNP (pg/ml) & $63.3(12.0-344.0)$ & $116.2(26.0-699.0)$ & $<0.0001$ \\
\hline Current smoking n, $(\%)$ & $97(7.4)$ & $7(6.5)$ & 0.70 \\
\hline Drinking $\mathrm{n},(\%)$ & & & 0.55 \\
\hline None & $91(7.0)$ & $6(5.7)$ & \\
\hline Occasional & $334(25.5)$ & $25(23.6)$ & \\
\hline Light & $625(47.8)$ & $51(48.1)$ & \\
\hline Moderate & $230(17.6)$ & $19(17.9)$ & \\
\hline Heavy & $26(2.0)$ & $5(4.7)$ & \\
\hline Physically inactive $\mathrm{n},(\%)$ & $66(5.0)$ & $6(5.6)$ & 0.80 \\
\hline $\begin{array}{l}\text { Previous myocardial infarction ] } \\
\mathrm{n},(\%)\end{array}$ & $117(8.9)$ & $10(9.3)$ & 0.70 \\
\hline Heart failure $n,(\%)$ & $11(0.8)$ & $1(0.9)$ & 0.86 \\
\hline Anti-hypertensive meds $\mathrm{n},(\%)$ & $305(23.4)$ & $33(30.6)$ & 0.092 \\
\hline
\end{tabular}

Values are number of subjects (\%), arithmetic mean \pm SD, or geometric mean (5th to 95 th percentile interval). ALT, alanine transaminase; AST, aspartate transaminase; NT-proBNP, $\mathrm{N}$-terminal prohormone of brain natriuretic peptide. 
Table 2 Associations between atrial fibrillation and baseline liver enzyme concentrations and changes in liver enzyme concentrations between baseline and follow-up

\begin{tabular}{llll}
\hline & \multicolumn{2}{c}{ Atrial fibrillation (Odds Ratio, 95\% CI) } \\
\hline Main independent variables & Unadjusted & +Age & Primary model \\
Baseline $\gamma$-glutamyl transferase (U/l) & $1.01(0.83-1.23)$ & $1.04(0.85-1.26)$ & $0.95(0.77-1.18)$ \\
$\Delta \gamma$-glutamyl transferase (U/l) & $1.22(1.05-1.41) \dagger$ & $1.21(1.04-1.40)^{*}$ & $1.18(1.01-1.37)^{*}$ \\
Baseline aspartate transaminase (U/l) & $0.89(0.72-1.09)$ & $0.92(0.75-1.12)$ & $0.89(0.72-1.10)$ \\
$\Delta$ Aspartate transaminase (U/l) (U/l) & $1.09(0.87-1.37)$ & $1.08(0.86-1.35)$ & $0.97(0.74-1.26)$ \\
Baseline alanine transaminase (U/l) & $0.84(0.69-1.03)$ & $0.91(0.74-1.12)$ & $0.85(0.69-1.06)$ \\
$\Delta$ Alanine transaminase (U/l) & $1.05(0.85-1.28)$ & $1.05(0.85-1.30)$ & $1.00(0.77-1.29)$ \\
Baseline alkaline phosphatase (U/l) & $0.57(0.16-2.06)$ & $1.13(0.93-1.36)$ & $1.11(0.92-1.35)$ \\
$\Delta$ Alkaline phosphatase (U/l) & $1.06(0.88-1.26)$ & $1.01(0.85-1.20)$ & $1.00(0.84-1.20)$
\end{tabular}

Primary model included baseline gamma-glutamyl transferase, age, body mass index, systolic blood pressure, diastolic blood pressure, total cholesterol, serum glucose, physical activity, smoking, drinking, use of anti-hypertensive medication, and follow-up time. $*, P<0.05 ; \dagger, P<0.01$. $\Delta$ denotes delta or change from baseline to follow-up. 
Table 3 Sensitivity analysis for the association between atrial fibrillation and gamma-glutamyl transferase

\section{Atrial fibrillation}

\begin{tabular}{|c|c|c|}
\hline$\Delta$ Gamma-glutamyl transferase (U/l) & OR $(95 \% \mathrm{CI})$ & $\boldsymbol{P}$ \\
\hline Primary model & $1.18(1.01-1.37)$ & 0.032 \\
\hline + baseline CRP & $1.20(1.03-1.40)$ & 0.018 \\
\hline$+\Delta \mathrm{CRP}$ & $1.20(1.03-1.39)$ & 0.020 \\
\hline + baseline ALT & $1.18(1.01-1.37)$ & 0.032 \\
\hline$+\Delta \mathrm{ALT}$ & $1.18(1.01-1.37)$ & 0.032 \\
\hline + baseline AST & $1.18(1.01-1.37)$ & 0.032 \\
\hline$+\Delta \mathrm{AST}$ & $1.18(1.02-1.38)$ & 0.031 \\
\hline +baseline ALP & $1.18(1.01-1.37)$ & 0.032 \\
\hline$+\triangle \mathrm{ALP}$ & $1.18(1.01-1.37)$ & 0.032 \\
\hline + AST/ALT ratio & $1.18(1.01-1.37)$ & 0.032 \\
\hline$+\Delta \mathrm{AST} / \mathrm{ALT}$ ratio & $1.18(1.02-1.38)$ & 0.031 \\
\hline + exclude GGT >61 U/1 & $1.44(1.18-1.76)$ & 0.0004 \\
\hline + exclude previous MI & $1.18(1.01-1.37)$ & 0.032 \\
\hline + exclude HF & $1.21(1.04-1.42)$ & 0.015 \\
\hline+ Baseline bilirubin & $1.18(1.01-1.37)$ & 0.032 \\
\hline$+\Delta$ bilirubin & $1.13(0.97-1.32)$ & 0.13 \\
\hline + Baseline NT-proBNP & $1.15(0.98-1.34)$ & 0.088 \\
\hline$+\Delta \mathrm{NT}$-proBNP & $1.08(0.91-1.28)$ & 0.40 \\
\hline$+\Delta$ NT-proBNP $+\Delta$ bilirubin & $1.05(0.88-1.25)$ & 0.62 \\
\hline
\end{tabular}

The primary model included baseline gamma-glutamyl transferase, age, body mass index, systolic blood pressure, diastolic blood pressure, total cholesterol, serum glucose, physical activity, smoking, drinking, use of anti-hypertensive medication, and follow-up time. ALT, alanine transaminase; AST, aspartate transaminase; CRP, C-reactive protein; GGT, gammaglutamyl transferase; HF, heart failure; MI, myocardial infarction; NT-proBNP, N-terminal prohormone of brain natriuretic peptide. $\Delta$ denotes delta or change from baseline to followup. 



Figure 1. Comparison of gamma-glutamyl transferase, N-terminal prohormone of brain natriuretic peptide (NT-proBNP), total bilirubin and haemoglobin at baseline and follow-up between those with and without atrial fibrillation at follow-up. AF, atrial fibrillation. 


\section{Liver enzymes are not directly involved in atrial fibrillation: a prospective cohort study}

Running head: Liver enzymes and atrial fibrillation

Rudolph Schutte, PhD ${ }^{\text {b,c,d; }}$; Peter H Whincup, MD, Ph D ${ }^{\text {e }}$; Olia Papacosta, MSc ${ }^{\text {a }}$, Lucy T Lennon, MSc ${ }^{\mathrm{a}}$; Peter W Macfarlane, DSc ${ }^{\mathrm{f}}$; Goya Wannamethee, $\mathrm{PhD}^{\mathrm{a}}$

${ }^{\mathrm{a}}$ Department of Primary Care \& Population Health, Institute of Epidemiology and Health Care, University College London Medical School, London, UK; ${ }^{b}$ Hypertension in Africa Research Team (HART), North-West University, Potchefstroom, South Africa; ${ }^{\mathrm{c}}$ South African Medical Research Council: Unit for Hypertension and Cardiovascular Disease, North-West University, Potchefstroom, South Africa; ${ }^{\mathrm{d}}$ Department of Medicine and Healthcare Science, Faculty of Medical Science, Anglia Ruskin University, Chelmsford, UK; e Population Health Research Institute, St George's, University of London, London, UK;

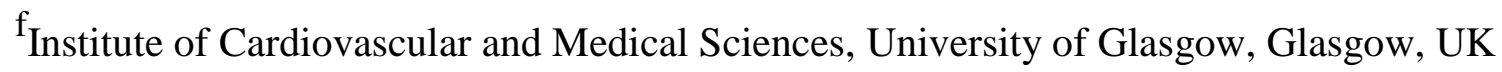
Supplementary tables 2 Supplementary figures 2 
Supplementary Table 1 Characteristics of men at follow-up with and without atrial fibrillation (AF)

\begin{tabular}{|c|c|c|c|}
\hline & $\begin{array}{c}\text { Without AF } \\
(n=1320)\end{array}$ & $\begin{array}{l}\text { With AF } \\
(n=108)\end{array}$ & $P$ \\
\hline Age (years) & $78.4 \pm 4.6$ & $80.1 \pm 5.2$ & 0.001 \\
\hline Body mass index $\left(\mathrm{kg} / \mathrm{m}^{2}\right)$ & $26.9 \pm 3.8$ & $27.4 \pm 3.9$ & 0.19 \\
\hline Systolic blood pressure (mmHg) & $146.0 \pm 19.2$ & $137.4 \pm 20.4$ & $<0.0001$ \\
\hline Diastolic blood pressure $(\mathrm{mmHg})$ & $76.2 \pm 11.3$ & $79.2 \pm 13.6$ & 0.028 \\
\hline Gamma-glutamyl transferase (U/l) & $24.4(11.0-69.0)$ & $33.2(14.0-115.0)$ & $<0.0001$ \\
\hline Aspartate transaminase (U/l) & $22.7(15.0-35.0)$ & $22.3(17.0-33.0)$ & 0.33 \\
\hline Alanine transaminase (U/l) & $18.6(10.0-35.0)$ & $18.4(11.0-34.0)$ & 0.75 \\
\hline Alkaline phosphatase (U/l) & $69.4(44.0-109.0)$ & $73.1(49.0-118.0)$ & 0.054 \\
\hline ALT:AST & $1.27 \pm 0.42$ & $1.34 \pm 0.45$ & 0.21 \\
\hline Total bilirubin $(\mu \mathrm{mol} / \mathrm{l})$ & $11.05(6.00-23.00)$ & $13.80(7.00-28.00)$ & $<0.0001$ \\
\hline Total protein $(\mathrm{g} / \mathrm{l})$ & $70.0 \pm 4.1$ & $69.9 \pm 4.1$ & 0.80 \\
\hline Serum albumin $(g / 1)$ & $44.8 \pm 2.4$ & $44.5 \pm 2.5$ & 0.15 \\
\hline Blood glucose $(\mathrm{mmol} / \mathrm{l})$ & $5.58(4.60-7.90)$ & $5.83(4.70-9.00)$ & 0.085 \\
\hline Total cholesterol (mmol/l) & $4.66 \pm 1.04$ & $4.39 \pm 0.98$ & 0.006 \\
\hline C-reactive protein $(\mathrm{mg} / \mathrm{l})$ & $1.30(0.18-9.82)$ & $1.96(0.42-14.76)$ & 0.0007 \\
\hline NT-proBNP (pg/ml) & $120(12-1057)$ & $993(264-3554)$ & $<0.0001$ \\
\hline Current smoking n, (\%) & $46(3.5)$ & $1(0.9)$ & 0.15 \\
\hline Drinking n, $(\%)$ & & & 0.62 \\
\hline None & $141(10.7)$ & $14(13.0)$ & \\
\hline Occasional & $401(30.4)$ & $34(31.5)$ & \\
\hline Light & $542(41.1)$ & $40(37.0)$ & \\
\hline Moderate & $152(11.5)$ & $17(15.7)$ & \\
\hline Heavy & $20(1.5)$ & $1(0.01)$ & \\
\hline Physically inactive $\mathrm{n},(\%)$ & $212(16.1)$ & $21(19.4)$ & 0.36 \\
\hline Previous myocardial infarction $\mathrm{n},(\%)$ & $135(10.2)$ & $11(10.2)$ & 0.70 \\
\hline Heart failure $n,(\%)$ & $61(4.6)$ & $13(12.0)$ & 0.0008 \\
\hline Anti-hypertensive meds $\mathrm{n},(\%)$ & $759(57.5)$ & $83(76.9)$ & $<0.0001$ \\
\hline
\end{tabular}

Values are number of subjects (\%), arithmetic mean \pm SD, or geometric mean (5th to 95 th percentile interval). AF, atrial fibrillation; AST, aspartate transaminase; ALT, alanine transaminase; NT-proBNP, N-terminal prohormone of brain natriuretic peptide. 
Supplementary Table 2 Univariate associations between change in NTproBNP and liver enzymes concentrations from baseline to follow-up Total group With AF Without AF

\begin{tabular}{lllllll}
\hline & \multicolumn{5}{c}{$\Delta$ NT-proBNP } \\
& $\mathrm{r}$ & $P$ & $\mathrm{r}$ & $P$ & $\mathrm{r}$ & $P$ \\
\hline$\Delta$ GGT & 0.14 & $<0.0001$ & 0.15 & 0.14 & 0.11 & $<0.0001$ \\
$\Delta$ ALT & 0.01 & 0.61 & 0.02 & 0.87 & 0.01 & 0.72 \\
$\Delta$ AST & 0.04 & 0.17 & 0.02 & 0.86 & 0.04 & 0.20 \\
$\Delta$ AST/ALP & -0.04 & 0.19 & -0.12 & 0.24 & -0.02 & 0.43 \\
$\Delta$ ALP & 0.10 & 0.0001 & 0.16 & 0.10 & 0.10 & 0.0005 \\
$\Delta$ Bilirubin & 0.13 & $<0.0001$ & 0.15 & 0.13 & 0.08 & 0.004
\end{tabular}

NT-proBNP, N-terminal prohormone of brain natriuretic peptide, GGT, gamma-glutamyl transferase; ALT, alanine transaminase; AST, aspartate transaminase; $\Delta$ denotes change from baseline to follow-up. 

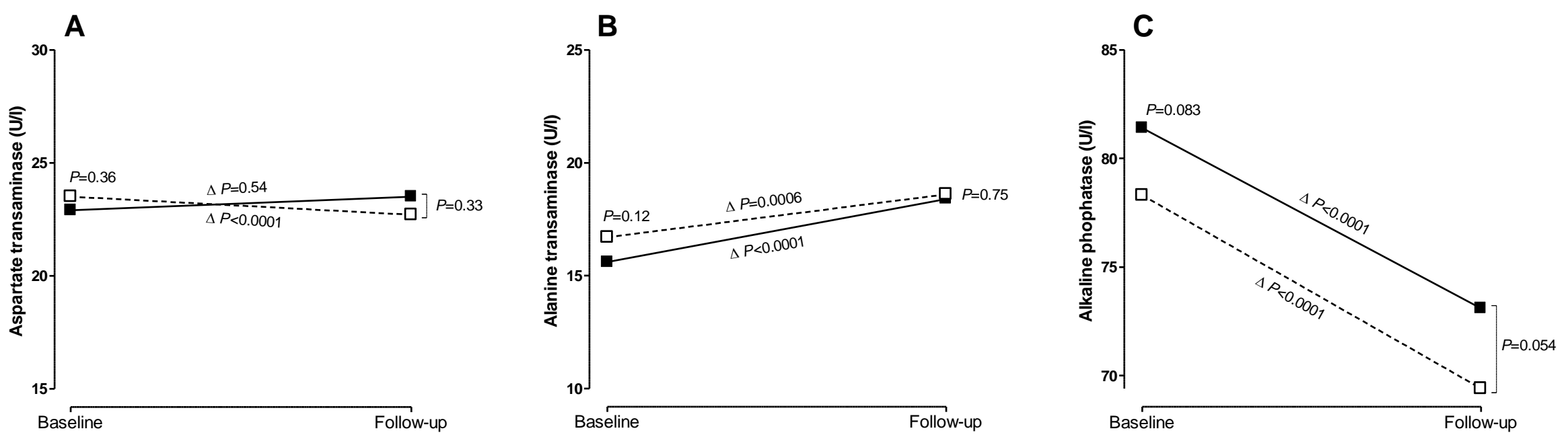

Supplementary Figure 1 Comparison of aspartate transaminase, alanine transaminase and alanine phosphatase at baseline and follow-up between those with and without atrial fibrillation at follow-up. AF. 


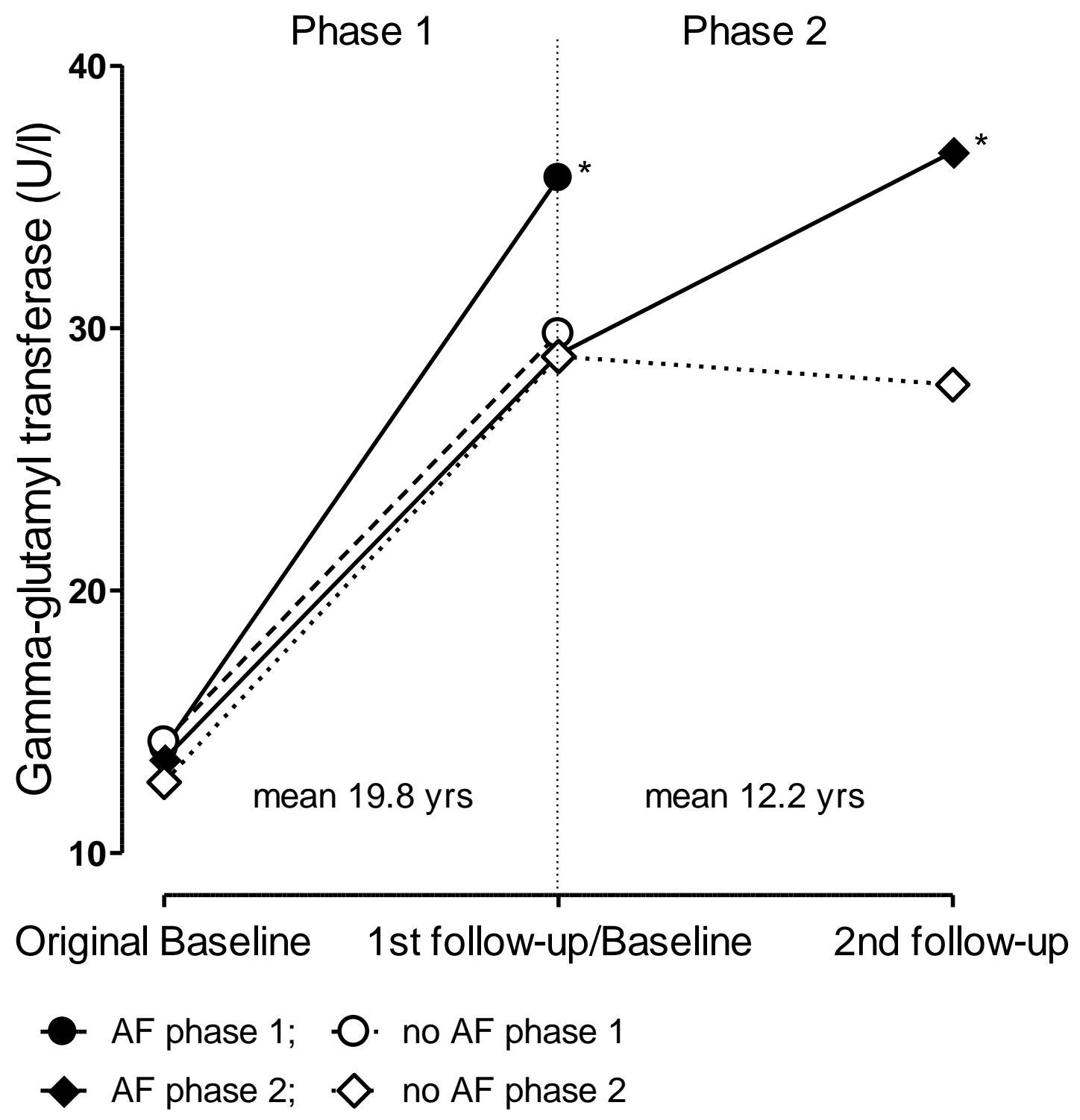

Supplementary Figure 2 Comparison of gamma-glutamyl transferase between those with and without atrial fibrillation at the original baseline (1978-1980), first follow-up/current baseline (1998-2000) and second follow-up (2010-2012). The circles represent participants with $(\bullet)$ and without $(\circ)$ atrial fibrillation that passed away after the first follow-up/current baseline (1998-2000) and not included in the present study. The diamonds represent the participants with $(\diamond)$ and without $(\diamond)$ atrial fibrillation included in the current study and alive at the second follow-up (2010-2012). 